\title{
Breve historia de la psicología en la ciudad de La Plata (1906-1966)*
}

\author{
A Brief History of Psychology in the City of La Plata (1906-1966)
}

Recibido: enero 26 de 2014 | Revisado: julio 26 de 2014 | Aceptado: julio 26 de 2014

\author{
AlejANDro A. DAgFal ** \\ Universidad de Buenos Aires, Argentina
}

doi:10.11144/Javeriana.upsy13-5.bhpc

Para citar este artículo: Dagfal, A. A. (2014). Breve historia de la psicología en la ciudad de La Plata (1906-1966). Universitas Psychologica, 13(5), 1759. 1775. http://dx.doi.org/10.11144/Javeriana.upsy13-5. bhpc

Este trabajo de investigación fue realizado en dos etapas. La primera, entre 1996 y 1998, fue financiada con una beca de iniciación en la investigación de la Universidad Nacional de La Plata. La segunda etapa, entre 2013 y 2014, fue financiada por el CONICET, y permitió sintetizar muchos de los datos recabados en la etapa anterior. Ciertos resultados parciales de la investigación de 19961998 circularon ilegalmente por Internet y fueron reproducidos por algunos historiadores.

** Consejo Nacional de Investigaciones Científicas y Técnicas (CONICET). Dirección postal: 68 № 720, La Plata (1900) Argentina. Dirección electrónica: adagfal@club-internet.fr

\section{RES U MEN}

Muy esquemáticamente, este trabajo aborda seis décadas de la historia de la psicología en la ciudad de La Plata (Argentina), que van desde la creación de la sección pedagógica en la Universidad Nacional, en 1906, hasta el golpe de estado de 1966, que puso fin al auge de los estudios psicológicos iniciado con la creación de la carrera profesional, en 1958. En ese período, la psicología pasaría por distintas etapas, signadas por oposiciones tales como "naturalismo positivista"/"humanismo antipositivista", "formación disciplinar"/"formación profesional", "psicología científica"/"psicología psicoanalítica", etc. El objetivo de este trabajo es dar cuenta de lo sucedido en ese lapso, poniendo el énfasis no solo en los actores individuales que intervinieron en ese proceso, sino también en los factores institucionales y políticos que conformaron el contexto. Los métodos utilizados son los tradicionales de la historia, básicamente, la recopilación de fuentes orales, documentales y bibliográficas, aunque se las haya interpretado desde una perspectiva crítica.

Palabras clave

historia; psicología; La Plata

\section{A B S T R A C T}

This paper takes very briefly on six decades of the history of psychology in the city of La Plata (Argentina), from the creation of the Pedagogic Department in the National University, in 1906, until the coup d'état of 1966, that put an end to the expansion of psychological studies that had started in 1958, after the creation of the psychology program. In that period, psychology would go through different phases, marked by oppositions such us "positivist naturalism"/ "anti-positivist humanism", "theoretical training" / "professional training", "scientific psychology" / "psychoanalytic psychology", etc. The goal of this work is to make an account of what happened at the time, giving as much importance to the individual actors as to the institutional and political factors that were present in the context. The methods are the traditional ones for history (mainly de gathering of oral, documentary and bibliographic sources. However, they have been interpreted from a critical perspective.

Keywords

history; psychology; La Plata 


\section{Introducción: psicología y educación, entre positivismo y antipositivismo}

Si bien la creación de la carrera de Psicología en la Universidad Nacional de La Plata (UNLP) recién se produjo en el año 1958, la disciplina ya tenía en esa ciudad una historia de más de cinco décadas. Prácticamente desde el nacimiento de la Universidad Nacional en 1905, la Psicología había ocupado un lugar central en el campo académico. En 1906, con la creación de la Sección Pedagógica de la Facultad de Ciencias Jurídicas (a cargo del célebre educador Víctor Mercante), se inició un período de casi veinte años durante el cual la psicología experimental aportó los fundamentos teóricos para una pedagogía que, dentro del marco del positivismo, aspiraba a ser científica. Si bien el objetivo de la Sección era la formación de profesores, el punto de aplicación de estas teorías eran los alumnos de las escuelas primarias, cuya educación debía basarse en normas generales que le aportaran racionalidad, y en determinados conocimientos prácticos. Tanto esas normas como esos conocimientos tenían que cimentarse de manera empírica, tarea a la que dedicarían gran parte de sus vidas el mismo Mercante, Rodolfo Senet y Alfredo Calcagno, privilegiando la utilización de métodos experimentales y estadísticos (Talak, 2007; Dagfal, 1996).

En 1914, durante el apogeo de este período, la Sección Pedagógica se convirtió en Facultad de Ciencias de la Educación, y su órgano de difusión (los Archivos de Pedagogía y Ciencias Afines) pasaría a llamarse Archivos de Ciencias de la Educación. Los vínculos con centros académicos del exterior parecían ser muy estrechos, si se considera la publicación de artículos de autores tales como Janet y Dumas y los relatos de Mercante sobre su visita al laboratorio de Claparède y su encuentro con Sante de Sanctis (Mercante, 1927). En 1920, jubilado Mercante, las "ciencias de la educación" (y por consiguiente la psicopedagogía), de la mano de una tardía declinación del positivismo, fueron desplazadas a un lugar menos central. La Facultad cambió su nombre por el de "Facultad de Humanidades" (y Ciencias de la Educación), adquiriendo mayor relevancia las secciones de filosofía e historia (Dagfal, 1996).
No obstante lo anterior, luego de su retiro, Mercante fue reemplazado en la cátedra de Psicopedagogía por su "sucesor natural": Alfredo Domingo Calcagno (1891-1962). Nacido en la ciudad de Mercedes, en la provincia de Buenos Aires, había cursado sus estudios en la Escuela Normal de esa ciudad, que por ese entonces estaba bajo la dirección del propio Mercante (quien, a su vez, se había formado en la Escuela Normal de Paraná, cuna del positivismo argentino). Cuando Mercante dejó la dirección de la Escuela Normal para hacerse cargo de la dirección de la flamante Sección Pedagógica de la UNLP, Calcagno siguió sus pasos, graduándose en esa institución en $1910^{1}$. Inducido por Mercante, obtuvo una beca de la Universidad para completar sus estudios en la Faculté Internationale de Paidologie, fundada en Bruselas por Josefa Joteyko. Allí obtuvo el título de Doctor en Ciencias Paidológicas, aunque debió regresar aceleradamente en 1914, en virtud de la guerra (Lunazzi, 1965).

A su regreso, comenzó su carrera docente en la recién creada Facultad de Ciencias de la Educación. Sin embargo, a diferencia de Mercante, además del estudio de los grupos escolares, Calcagno iba a privilegiar la experimentación de laboratorio desde una perspectiva más afín a la tradición alemana. Por este motivo, también se afanó por modernizar el Laboratorio de Psicopedagogía fundado por su maestro, agregando aparatos de medición que él mismo había inventado o había hecho traer al país (Calcagno, 1921; Mercante, 1927). Con el paso del tiempo, en un clima cada vez más hostil a esta concepción experimental de la psicología -que Calcagno veía como un "capítulo especial" de la fisiología-, prefirió consagrarse de lleno a la labor política, siendo elegido decano de la Facultad en $1934^{2}$.

Durante esa década, a excepción de Enrique Mouchet (Klappenbach, 2001), no habría en la Facultad figuras muy destacadas por su producción

1 Según Lunazzi, Calcagno también habría sido cofundador de la Federación Universitaria de La Plata. No obstante, sitúa esa fundación en 1916. Hugo Biagini, por su parte, la ubica en 1911, con una posterior reorganización en 1917 (Lunazzi, 1965; Biagini, 1995).

2 Más adelante sería elegido presidente de la Universidad, en 1944, y diputado nacional por la Unión Cívica Radical, en 1946. 
en el campo de la psicología, ya que, en esa época, Calcagno sobresalía más por su actividad institucional que por su elaboración teórica. Paralelamente, la disciplina se emparentaba con posiciones más filosóficas, acusando la recepción de autores como Dilthey, Spranger y Bergson. Los dos primeros habían introducido la significación como un tema central para las ciencias del hombre, mientras que el tercero había asestado un duro golpe a la psicología experimental, ensalzando la intuición y la experiencia subjetiva a partir de argumentos que encontraron un público muy ávido de nuevos saberes.

En la década de los cuarenta, la Facultad ya constituía un espacio muy complejo y heterogéneo donde se daban cita numerosos profesores de extracción diversa, quienes poseían un cierto renombre o lo adquirirían en años subsiguientes. En la Sección de Filosofía y Ciencias de la Educación dictaban clases, en 1945, Coriolano Alberini y Eugenio Pucciarelli (en Introducción a la Filosofía), Luis Juan Guerrero (en Estética), Marcos Victoria (en Biología del Sistema Nervioso), Ernesto Figueroa (en Psicología), Francisco Romero (en Lógica), Carlos Astrada (en Ética), Alfredo Calcagno (en Psicopedagogía) y Alberto Palcos (en Teoría e Historia de las Ciencias); mientras que en la Sección de Historia y Geografía también daban clases Ricardo Levene, José Luis Romero, Vicente Fatone y Abraham Rosenvasser (“Cuerpo docente”, 1945, pp. 236-237). Como muestra de la pluralidad teórica puede notarse que convivían en una misma institución el continuador de la tradición experimental y positivista de Víctor Mercante (Alfredo Calcagno), los introductores del novecentismo antipositivista y del pensamiento "espiritualista" de Bergson (Alberini y Figueroa), junto con un médico neurólogo formado con Christofredo Jakob (Marcos Victoria), quien sería más adelante el primer director de la carrera de psicología de la Universidad de Buenos Aires (UBA).

\section{El período peronista: psicología aplicada y orientación profesional}

El primer peronismo (1946-1955) implicó un punto de quiebre para la tradición académica de la UNLP.
Numerosos profesores abandonaron sus cargos y muchos otros fueron exonerados. La Universidad Reformista, que se identificaba como autónoma y laica, pasó a depender de manera directa del poder político. Los sectores más conservadores de la Iglesia llegaron a detentar posiciones importantes, en particular en la Facultad de Humanidades y Ciencias de la Educación, donde adquirieron un impensado relieve figuras tales como los presbíteros Octavio Derisi (quien fue el primer director del Instituto de Filosofía), Guillermo Blanco (profesor interino de Psicología) y Juan Sepich (profesor titular de Ética, que 1949 tendría un rol fundamental en la organización del Primer Congreso Nacional de Filosofía). Sin embargo, la inspiración aristotélicotomista de estos sacerdotes no dejaba de admitir, previa resignificación, el ingreso de corrientes de pensamiento como el existencialismo, dando lugar a una especie de "humanismo cristiano" que trascendería más allá de la caída de Perón (Piñeda, 2006). Mientras tanto, profesores como Eugenio Pucciarelli y Luis Felipe García de Onrubia (titular de Psicología entre 1947 y 1949), que habían sido alumnos de Korn y Alberini, introducían la fenomenología y la Gestalttheorie [Teoría de la Gestalt].

Resulta claro que, durante este período, la psicología académica era eminentemente teórica. Sin embargo, a partir del Estado provincial (y notoriamente al margen de cualquier vinculación con la Universidad), se desarrollaría una psicología aplicada dentro del campo de la educación, no ya con fines pedagógicos, sino con la intención de vincular la escuela con el mercado de trabajo a través de la orientación profesional. Con este fin se creó en 1948 el Instituto de Psicología Educacional y Orientación Profesional, como dependencia de la Dirección General de Escuelas. Allí se incorporaron, en carácter de "técnicos", el Dr. Bernardo Serebrinsky (un conocido psiquiatra infantil del grupo de Gregorio Bermann ${ }^{3}$ ) y Jaime Bernstein (socio fundador de la editorial Paidós, dedicado a la psicometría y los problemas de la educación, que

3 Bermann sería un personaje clave en la organización del campo de la psiquiatría a nivel nacional. 
luego sería un personaje clave en los inicios de la carrera de Psicología en Rosario) ${ }^{4}$.

En 1949, el Instituto fue elevado al rango de Dirección Provincial, quedando a cargo de Ricardo Moreno (quien años después sería uno de los secretarios del Primer Congreso Argentino de Psicología). A partir de una psicología actualizada por la adopción de métodos estadísticos y el empleo de test psicométricos y proyectivos, se buscaba clasificar a los alumnos del último año de la escuela primaria según sus aptitudes, condiciones físicas e inteligencia para poder realizar un "consejo profesional" adecuado. Esta tarea implicaba una optimización del aprovechamiento de los recursos humanos en el trabajo, por lo que había sido jerarquizada a partir de la industrialización promovida por el peronismo, mereciendo incluso un párrafo en la Constitución Nacional de 1949 (Klappenbach, 2001; Munín, 1989).

El Estado se constituía así en el principal promotor de una psicología aplicada, que no se agotaba en la orientación profesional, sino que incluía tareas de "asistencia", más vinculadas con la orientación psicopedagógica y con la detección y atención de los "niños-problema" (función que años más tarde se denominaría "clínica de conducta"). Para tal fin, se creó la figura del "asistente educacional" y se implementó (aunque de manera bastante asistemática) una suerte de escuela para "técnicos en psicología", destinada a la formación de los maestros. Estos maestros, devenidos asistentes educacionales, fueron la punta de lanza de la psicología en el sistema

4 En los años treinta, Serebrinsky había sido miembro del comité de redacción de la revista Psicoterapia, fundada por Bermann. Durante la Guerra Civil, lo acompañó en su aventura española, junto al bando republicano. En los años cuarenta, dictó conferencias en la Sociedad de Psicología y publicó diversos artículos en la Revista de Psiquiatría. En 1945, tradujo y prologó la primera versión castellana de Diagnóstico del desarrollo normal y anormal del niño, de Arnold Gesell y Catherine Amatruda, para la editorial Paidós, que acababa de ser creada justamente por Bernstein y Enrique Butelman. Jaime Bernstein, a su vez, había obtenido su título de pedagogo en 1942, en la Facultad de Filosofía y Letras de la UBA. Su tesis, intitulada "Psicología y educación adlerianas", preanunciaba sus elecciones teóricas futuras. Entre 1941 y 1942, Bernstein había formado parte del Instituto Sigmund Freud, fundado por el psicólogo húngaro Bela Székely. Como "subjefe de la sección pedagógica", había impartido cursos sobre el test de Rorschach y la inteligencia infantil, iniciando una "actividad clínica de orientación educacional” (Dagfal, 2009). educativo. Intervenían en el seno de la escuela autorizándose en un saber técnico novedoso, que aún no gozaba de demasiado prestigio entre las docentes más tradicionales. Más allá de las palabras pomposas de los funcionarios políticos, cumplieron un rol muy importante en la difusión de la psicología en el campo de la educación, si se considera que, solo en el año 1948, se expidieron 1857 consejos profesionales (sobre un total de 2600 alumnos de sexto grado del distrito La Plata) $)^{5}$. Cada consejo profesional implicaba un largo proceso, durante el cual el asistente educacional debía entrevistar a los alumnos, a sus padres y a sus maestros, con lo que la cantidad de actores involucrados se multiplicaba ${ }^{6}$. La recepción de la comunidad para estos nuevos personajes fue positiva o, en todo caso, tibiamente indiferente.

En 1954, con la organización del Primer Congreso Argentino de Psicología en San Miguel de Tucumán, se produjo uno de los antecedentes más importantes para la creación de las carreras de Psicología en el país (Gentile, 2003). Allí se dieron cita por primera vez profesores de psicología y practicantes de origen diverso, incluyendo filósofos, psicoanalistas, psiquiatras, pedagogos y psicotécnicos. También asistieron algunos invitados extranjeros, como Pascual de Roncal y René Lacroze, y mandaron comunicaciones personalidades como Gordon Allport y Agostino Gemelli, entre otros. Ricardo Moreno, que había sido relevado de su cargo en la Dirección en 1952 y ya era profesor en Tucumán, fue designado secretario del congreso (junto a Oscar Oñativia). Por su parte, Francisco González Ríos (profesor de la Facultad de Humanidades en el primer curso de Psicología), fue elegido representante de los delegados nacionales, y en ese carácter pronunció el discurso de apertura. En la sesión plenaria realizada en la ciudad de Salta el 22 de marzo se aprobó una declaración que afirmaba

5 El objetivo final era relevar los alumnos de sexto grado de toda la provincia, para lo cual, en 1950, se crearon las primeras filiales de la Dirección en ciudades del interior de la provincia, como Bahía Blanca, Avellaneda y Azul (Munín, 1989, p. 108).

6 Incluso los maestros - por lo general mujeres- eran capacitados para llenar una ficha escolar anual, en la que debían volcar información sobre diversos aspectos psicológicos de todos sus alumnos. 
"la necesidad" de crear la carrera universitaria del "psicólogo profesional" [...], "como sección autónoma en las facultades de carácter humanístico, aprovechando los institutos ya existentes".

Esa declaración había sido presentada por los profesores Luis Juan Guerrero, Eugenio Pucciarelli, Alberto Palcos, Francisco González Ríos, Carlos Astrada, Ricardo Moreno, Oscar Oñativia, Plácido Horas, Luis María Ravagnan y Osmán Dick. Cabe destacar que, de esos diez profesores, cinco eran docentes de la UNLP o lo habían sido recientemente (Guerrero, Pucciarelli, Astrada, Palcos y González Ríos), uno había estado a cargo de la Dirección de Psicología (Moreno) y otro sería docente de la UNLP y Director de la Dirección de Psicología (Ravagnan) ${ }^{7}$. Todo parecía indicar que este grupo heterogéneo, compuesto sobe todo por filósofos y pedagogos de orientaciones muy diversas, había arribado a los consensos necesarios para peticionar por la creación de la carrera universitaria de Psicología de manera efectiva, como lo demuestra el hecho de que pocos años después muchos de sus miembros ocupasen lugares destacados en la apertura de las carreras de La Plata, San Luis, Tucumán, Rosario y, en menor medida, Buenos Aires.

Semanas más tarde, el profesor González Ríos presentó ante el Consejo Directivo de la Facultad de Humanidades y Ciencias de la Educación de la UNLP un proyecto proponiendo la creación de la carrera y el Instituto de Psicología (Facultad de Humanidades y Ciencias de la Educación, 1954, p. 70). La carrera no fue aprobada, pero sí el Instituto "por así exigirlo el profundo proceso de realización en lo económico, en lo político y en lo social que actualmente se cumple en el país" (Universidad Nacional de La Plata, 1954) ${ }^{8}$. El profesor González

7 Según el testimonio personal de Ricardo Moreno, otro de los signatarios, Plácido Horas, había viajado expresamente a La Plata para estudiar la organización de la Dirección a su cargo, creando al regresar a San Luis una Dirección homónima, por convenio entre la Universidad de Cuyo y el gobierno provincial (Moreno, 1997).

8 De todos modos, no se han encontrado evidencias de ninguna actividad concreta de dicho instituto hasta 1957. Por otra parte, en la Universidad Nacional del Litoral (en la ciudad de Rosario), el Instituto de Psicología se creó en mayo de 1954, también en estrecha relación con el discurso oficial y los planes de gobierno. Hacia fin de año, el Instituto elevó al Consejo Directivo el Pro-
Ríos fue nombrado director del Instituto, que a su vez pasó a depender del Departamento de Filosofía y Pedagogía.

\section{La caída de Perón, el Estado provincial y la "normalización" de la Universidad Nacional de La Plata}

En 1955, el derrocamiento de Perón implicó un cambio radical en el panorama político, no exento de matices (Dagfal, 2012). La dictadura militar que tomó el poder (autodenominada "Revolución Libertadora") tomó drásticas medidas represivas, que afectaron particularmente a los sectores populares que habían apoyado al "tirano depuesto", cuyo nombre ya ni siquiera podía ser pronunciado. Al mismo tiempo, para las clases más favorecidas, 1955 marcó el inicio de un acelerado período de renovación social y cultural, similar al vivido por las naciones europeas durante la segunda posguerra. La ciudad de La Plata recuperaba su antiguo nombre y dejaba de llamarse "Eva Perón". El populismo tradicionalista del gobierno derrocado fue rápidamente reemplazado por el ideario liberal de la dictadura triunfante. Eran tiempos de modernización y apertura, que preanunciaban lo que iba a suceder en la década del sesenta.

El Estado provincial intervenía activamente en el campo cultural a través de diversas iniciativas. El Ministerio de Educación, por ejemplo, comenzó a publicar "la nueva serie" de la Revista de Educación (heredera de los Anales fundados por Domingo Faustino Sarmiento casi cien años antes). Incluía una sección de "artículos" (originales), otra de "estudios y traducciones", una de "actualidad pedagógica", otra de "lecturas", una de "lenguaje y estilo" y, finalmente, una dedicada a reseñas de libros de reciente aparición. Su misma estructura ya dejaba trasuntar una voluntad de amplitud en la convivencia de distintos saberes, receptando

yecto de la carrera de psicólogo. El 6 de abril de 1955, el Consejo Superior aprobó en Santa Fe la creación de la primera carrera universitaria de psicología del país. Esta carrera tendría corta vida, ya que luego del golpe del 16 de septiembre debió "reorganizarse" el plan de estudios, por lo que las clases no se reanudaron hasta el año siguiente, con un nuevo plan, aprobado en febrero del 56. 
ávidamente el existencialismo (en sus vertientes más "optimistas", con artículos de Gabriel Marcel y Jean Wahl) e incluso a las corrientes epistemológicas "no racionalistas" (con traducciones de Gaston Bachelard y George Canguilhem, entre otros) ${ }^{9}$. También tenían un lugar las últimas tendencias de la sociología "científica" y las diversas psicoterapias. El lugar de privilegio otorgado por la revista a la disciplina psicológica merece un párrafo aparte. A tono con la popularización masiva de la psicología que se venía registrando desde la década anterior, ya en los primeros números se incluían referencias a psicólogos y psiquiatras europeos (como Emilio Mira y López, Paul Fraisse y Daniel Lagache), latinoamericanos (como Honorio Delgado y Alberto Seguin) y argentinos (como Luis María Ravagnan, Plácido Horas y Florencio Escardó) ${ }^{10}$.

La Dirección de Psicología (mencionada más arriba), continuaba con sus actividades, pero bajo un nuevo nombre: "Dirección de Psicología Educacional y Asistencia Social Escolar". Llaman la atención las palabras del interventor en un informe que respondía a ciertas acusaciones que se habían planteado ante el Ministerio:

En lo que se refiere a la imputación de orden técnico, referente a que se utilizaban métodos psicoanalíticos para el tratamiento psicológico de menores de edad, especialmente púberes, ocurre otro tanto, ya que la preparación del personal, así como el examen de los expedientes han probado una amplia heterodoxia respecto de la doctrina mencionada. (Munín, 1989, pp. 119-120)

Esta necesidad de demostrar que no se había "incurrido" en la utilización de métodos psicoanalíticos muestra que, pese a lo que podían indicar las apariencias (si uno se guiara por la línea sostenida por la Revista de Educación), la recepción del psi-

9 Es notoria la celeridad con que eran traducidos algunos autores europeos. Por caso, la conferencia de Canguilhem "¿Qué es la psicología?", pronunciada en 1956, fue publicada en el año 1958 (pocas semanas después de su publicación en Francia).

10 Este último, médico pediatra del Hospital de Niños, tendría un importante papel en la difusión de las ideas psicológicas en los años por venir, a partir de sus columnas en el diario La Razón y la revista Primera Plana. coanálisis en las reparticiones del Estado no había sido tan sencilla. En todo caso, este ejemplo sirve para matizar el lugar común historiográfico según el cual el peronismo habría sido un acérrimo opositor del freudismo, mientras que el régimen que lo sucedió lo habría recibido con los brazos abiertos y lo habría promovido sin fisuras.

Por su parte, en la Universidad, la llegada del nuevo gobierno militar fue saludada con júbilo por los sectores universitarios platenses, con una larga tradición laica y liberal. La intervención había sido vivida de manera traumática, en particular por las organizaciones estudiantiles que, desde la clandestinidad, se habían transformado en pilares de la resistencia. Luego de la caída de Perón, reclamaron a través de los centros y de sus representantes un espacio acorde con el rol protagónico que habían desempeñado. Se erigieron en custodios del legado reformista, enarbolado los históricos principios de la autonomía, la libertad de cátedra y el gobierno tripartito.

En ese marco, el período 1955-1957, denominado "de normalización", implicó una acelerada reconstitución de los claustros (con la reincorporación de numerosos docentes cesanteados, como José Luis Romero, Alfredo Calcagno, etc.) que desembocaría en noviembre de 1957 en la elección de autoridades según los principios de la reforma. El clima general era de "recuperación democrática", de renovadas esperanzas en el futuro y, por consiguiente, de una clara vocación participativa.

\section{Bernardo Canal Feijóo, Alfredo Calcagno y Fernanda Monasterio: artífices de la creación de la carrera}

En la Facultad de Humanidades y Ciencias de la Educación, el encargado de conducir ese proceso de "normalización" fue el decano interventor, Bernardo Canal Feijóo. No obstante, antes de dejar su lugar al decano electo, Abraham Rossenvaser (un conocido historiador y antropólogo), Canal Feijóo fomentó la introducción de numerosos cambios. Por un lado, se modificaron los planes de estudios y se amplió la oferta de materias de filosofía y psicología. Por el otro, en mayo de 1957, el decano convocó a 
una comisión especial encargada de proponer un plan de estudios para un profesorado en psicología que aún debía crearse (Facultad de Humanidades y Ciencias de la Educación, 1958, p. 17-18). Según veremos más adelante, esta comisión terminaría yendo más allá de sus funciones, y propondría además la creación de una carrera profesional y un doctorado en psicología. No obstante, habría que esclarecer los motivos que llevaron a este decano interventor a promover tan activamente los estudios universitarios de psicología, para lo cual nos detendremos en su recorrido.

Lo menos que podría decirse es que Bernardo Canal Feijóo (1897-1982) era un personaje multifacético: poeta, ensayista, dramaturgo, historiador, jurista, sociólogo y folclorista, sus múltiples ocupaciones le valieron un reconocimiento extendido. Ya en 1934, obtuvo la Legión de Honor del Gobierno de Francia por la traducción de la obra capital de los hermanos Emile y Duncan Wagner, considerados fundadores de la arqueología santiagueña (Wagner \& Wagner, 1934). En 1975, llegaría a ser miembro de la Academia Argentina de Letras, de la que era presidente en el momento de su muerte (Tasso, 1997; Vallejo, 2008). Oriundo de Santiago del Estero, había cursado sus estudios universitarios en Buenos Aires, donde se recibió de abogado en 1918 y de doctor en jurisprudencia en 1922. Durante esa época, se vinculó con las vanguardias literarias, particularmente con el grupo de la revista Martín Fierro, renovador del verso y la composición. Al mismo tiempo, se acercó a los sectores reformistas, llegando incluso a ser amigo de Deodoro Roca, Gregorio Bermann y los hermanos Jorge y Arturo Orgaz $z^{11}$.

De vuelta en Santiago del Estero, en donde ejerció como abogado, participó en la creación del grupo "La Brasa", una entidad en la que se dieron cita escritores, artistas e intelectuales. Esta institución revolucionó el paisaje cultural local, publicando una revista y convocando a invitados de renombre, desde pintores argentinos como Antonio Berni y Emilio Petorutti hasta ilustres escritores extran-

11 Jorge Orgaz ocuparía un rol importante en la creación de la carrera de psicología en Córdoba. jeros como el conde de Keyserling y Pierre Drieu La Rochelle. En los años treinta, antes de empezar a publicar en la revista Sur, Canal se abocó a un profundo análisis del "ser santiagueño", que se vio reflejado en numerosos ensayos sobre el arte, los mitos y otras expresiones populares regionales ( $\mathrm{Ca}$ nal Feijóo, 1934, 1937, 1938, 1943). Reinstalado en Buenos Aires, a partir de 1946, se dedicó más bien a interpretar la realidad argentina desde un punto de vista sociológico y jurídico (Canal Feijóo, 1951, 1955), mientras que su correspondencia incluía a personajes tales como Eduardo Mallea, Macedonio Fernández, Marcos Victoria, Juan Filloy, Eugenio Pucciarelli y Atahualpa Yupanqui (Vallejo, 2008). Con estas credenciales, luego de la caída del peronismo (del cual había sido un opositor acérrimo) era más que comprensible que fuera llamado a ocupar cargos destacados en una universidad reformista signada por el antiperonismo.

Pero hay un aspecto de la obra de este autor que había sido apenas destacado hasta hace poco tiempo y que es susceptible de iluminar su interés por la creación de la carrera. Nos referimos a lo que Segundo Pablo Vallejo ha dado en llamar "el psicoanálisis de Bernardo Canal Feijóo". En efecto, a partir de su interés por la antropología, este abogado santiagueño interpretó mitos y leyendas populares en clave psicoanalítica, valiéndose principalmente del texto Tótem y tabú, y poniendo de relieve el problema del incesto. Para Canal, las producciones culturales encerraban un sentido oculto, ligado a los orígenes y a la identidad, que el investigador debía restituir a partir de detalles y fragmentos. $\mathrm{Al}$ mismo tiempo, en un contexto en el que el monte santiagueño desaparecía vertiginosamente ante el avance modernizador, su interés por el folclore aparecía a la vez como un contraveneno para los males de la civilización y como una vía para reencontrarse con un pasado pleno de sentido, opuesto a la idea de desierto, tan cara al positivismo vernáculo. Sin embargo, Vallejo muestra bien que para Canal no se trataba de reconstituir un pasado histórico sobre bases empíricas. Más que historizar, buscaba un origen cuasimítico, como el Freud de Totem y tabú (o los hermanos Wagner de La civilización chaco-santiagueña). Y este interés ya quedaba de 
manifiesto en sus textos de los años treinta, como Mitos perdidos (1938), en los que la literatura trágica popular (particularmente la "leyenda del Kakuy", que también había captado la atención de Ricardo Rojas) era la ocasión para desplegar toda su tarea interpretativa. Con estos elementos en vista, puede entenderse mejor por qué Bernardo Canal Feijóo terminó siendo un ferviente impulsor de la creación de la carrera de psicología en una Facultad de Humanidades.

En ese marco, cuando en 1957 Canal Feijóo convocó una comisión especial para diseñar un plan de estudios para el profesorado de psicología, eligió como presidente a Alfredo Domingo Calcagno, quien terminaría constituyéndose en el mayor promotor institucional de la carrera. Calcagno, cuya formación ya hemos situado en relación con el período positivista y la ascendencia de Víctor Mercante, había retornado a la UNLP en 1955, como director del Departamento de Ciencias de la Educación de la Facultad. Durante el período anterior, poco después de haber sido elegido presidente de la UNLP, había sido expulsado. No obstante, había continuado la guerra por otros medios, combinando sus actividades políticas en el Congreso de la $\mathrm{Na}$ ción con sus tareas intelectuales como director de la colección "Biblioteca de Ciencias de la Educación", de la editorial Kapelusz ${ }^{12}$.

Considerando las trayectorias de Bernardo Canal Feijóo y Alfredo Calcagno, podría pensarse que, en cierto modo, la conformación heterogénea que tendría la comisión especial no haría más que reflejar las formas radicalmente divergentes en que ambos concebían la psicología. Esquemáticamente, siguiendo a Foucault, podría decirse que el primero la consideraba una disciplina del sentido, ligada al psicoanálisis, las ciencias sociales y las humanidades. El segundo, por el contrario, la veía como una ciencia natural, emparentada con la biología y los métodos experimentales. Así, considerando el peso institucional de estas dos figuras, es entendible que los otros cuatro miembros de la comisión espacial

12 En 1947, Calcagno iba a liderar la oposición parlamentaria al proyecto oficial de ley universitaria (Pronko, 1995; García Sebastiani, 2005). se hayan repartido de manera salomónica entre dos vertientes. Por un lado, Ángel Garma y Zubizarreta (1904-1993) y Luis María Ravagnan (1902-1984), cercanos al enfoque humanista de Canal Feijóo; por el otro, Fernanda Monasterio Cobelo (1920-2006) y Juan Cuatrecasas Arumí (1899-1990), afines al enfoque naturalista de Calcagno.

Garma era un célebre psiquiatra bilbaíno, más conocido por haber sido cofundador y primer presidente de la Asociación Psicoanalítica Argentina (APA), en 1942. Desde 1957, dictaba en la Facultad un programa de Psicología General, de orientación claramente analítica (Dagfal, 2009). Ravagnan, un filósofo admirador de Merleau-Ponty, era profesor adjunto de esa misma materia, en la que impartía las unidades ligadas a la fenomenología y el existencialismo (Klappenbach, 2001). Por otra parte, Monasterio era una médica madrileña, que había sido discípula y colaboradora del célebre endocrinólogo Gregorio Marañón. Se había formado también en psicología general con José Germain (en el Instituto Nacional de Psicología y Psicotecnia) y en orientación profesional, con José Mallart (Quintana \& Feldman, 2007). En 1956, Calcagno la había convocado para dirigir el Instituto de Psicología y para dictar la materia Psicología de la Infancia y de la Adolescencia, perteneciente al Departamento de Ciencias de la Educación (Dagfal, 2009). Al igual que Calcagno, entendía la psicología como una ciencia natural, y ambos tenían una pasión común por el tema del desarrollo y el paradigma evoluti$\mathrm{vo}^{13}$. En consecuencia, con esas credenciales, pese a su relativa juventud (tenía entonces treinta y siete años) no resulta extraño que hubiera sido incluida en esta comisión ${ }^{14}$. En cuanto a Cuatrecasas, era un médico catalán, nacido en Camprodón (Gerona). Discípulo de Augusto Pi y Suñer (creador de la Escuela de Fisiología de Barcelona) emigró a la

13 El mismo Calcagno, según el modelo ribotiano había emprendido estudios de medicina, que luego dejó inconclusos. A su vez, había organizado y dirigido la edición argentina del Tratado de psicología de George Dumas (Lunazzi, 1965).

14 Monasterio había emigrado de España en 1952. Había estado en Bolivia, en la Universidad de Cochabamba, dictando fisiología; estuvo luego en Mendoza, en la Universidad de Cuyo, a acrgo de diversas asignaturas hasta 1956. En ese año asumió como titular en la cátedra de Psicología en la Universidad del Sur. 
Argentina en 1936, a causa de la guerra civil. Considerando la filogenia y la ontogenia, afirmaba que la conducta y el psiquismo dependían del grado de evolución de las estructuras perceptivas. En razón de estas ideas, gustaba de ser llamado "psicobiólogo" (Kurowsky, 2001).

En resumen, la "comisión especial" estaba conformada por cinco miembros con trayectorias bastante dispares en pedagogía, filosofía, medicina y psicoanálisis, disciplinas que en mayor o menor medida también estuvieron presentes en los núcleos iniciales de otras carreras de psicología del país (como las de Rosario, Buenos Aires, San Luis y Tucumán). Curiosamente, la mayoría (tres de ellos) eran médicos españoles emigrados, que reconocían la influencia de Gregorio Marañón (Carpintero, 2012). A excepción de Garma (cuyo soporte institucional era la APA y dedicaba la mayor parte de su tiempo a la atención de pacientes) y Monasterio (que ocasionalmente había ejercido la psicoterapia y había realizado tareas de orientación profesional) los otros integrantes tenían un perfil eminentemente académico, centrado en la universidad y alejado de eventuales prácticas profesionales.

Del profesorado en psicología a la carrera de psicología

La "comisión especial" se reunió con cierta periodicidad durante algunos meses. Finalmente, Alfredo Calcagno y Fernanda Monasterio terminaron redactando los proyectos para presentar al Consejo Directivo de la Facultad. No obstante, en los proyectos que se elevaron al Consejo Directivo en marzo de 1958 había una novedad: ya no se trataba de un "profesorado en psicología", según el encargo original del decano Canal Feijoo, sino de una "carrera de psicólogo".

En el medio, habían incidido factores que iban más allá del plano personal o biográfico. En febrero, la Asociación de Estudiantes del Instituto de Perfeccionamiento Docente (que dependía a la vez del Departamento de Ciencias de la Educación y del Ministerio de Educación) había elevado al rectorado un pedido formal para que se abriera la carrera de psicología. Sin proponérselo, el mismo Estado provincial había generado un polo de demandas de estudios psicológicos en el seno del sistema educativo. Muchos de los asistentes educacionales formados por el Ministerio para intervenir en las escuelas (mujeres en su mayor parte), reclamaban continuar sus estudios en una carrera mayor que tuviera una salida profesional jerarquizada. A su vez, los alumnos de Ciencias de la Educación veían en la psicología una posibilidad de ampliar sus horizontes laborales en una dirección radicalmente distinta de las ofrecidas por los profesorados de la Facultad.

La renovación social y cultural -de la que ya hemos hablado más arriba-colocaba a la psicología (y al psicoanálisis) en un lugar de preferencia. Era la clave que permitía dar significación a los vertiginosos cambios que la modernización traía aparejados en el tejido social y, en particular, en el seno de la familia. Lo que ocurría en Buenos Aires no podía sino tener consecuencias en La Plata, ubicada a apenas una hora de distancia. Allí, a partir de la creación de la APA (con su Revista de Psicoanálisis y su impacto en las élites intelectuales) y de la difusión a gran escala de la psicología (a través de la televisión, de revistas de distinta clase y de libros publicados por editoriales como Paidós y Kapelusz, que eran ávidamente consumidos por el público), los saberes "psi" habían terminado por arraigar fuertemente en la cultura (Balán, 1991; Plotkin, 2003; Terán, 1991; Vezzetti, 1996). Por otra parte, los estudiantes habían desempeñado un papel muy activo en la apertura de la carrera de psicología de la UBA (acorde con el protagonismo penosamente obtenido durante las épocas de la resistencia), por lo que en La Plata, la apertura de la carrera tampoco tardó en transformarse en una causa de militancia política defendida por el Centro de Estudiantes y la Federación Universitaria.

Del mismo modo, en el cuerpo de profesores, había varios docentes que dictaban clases en Filosofía y Letras de la UBA y en Humanidades de la UNLP. Entre ellos, los más influyentes eran Marcos Victoria (que por entonces había renunciado para hacerse cargo de la dirección de la carrera de psicología de la UBA) y Francisco Romero (renombrado filósofo, hermano del rector de la UBA, José Luis Romero). A todo esto debe sumarse el hecho de que en noviembre de 1957 se había completado el proceso de normalización de la Universidad, eligiéndose 
autoridades por el voto de los tres claustros. El nuevo decano de la Facultad, Abraham Rossenvaser, un conocido historiador y antropólogo, se transformó en uno de los más entusiastas impulsores del proyecto. En resumen, la iniciativa de crear la carrera de psicología terminó siendo patrimonio de un sujeto colectivo muy heterogéneo, que nucleaba a diversos actores que la reclamaron con insistencia desde distintas posiciones y a partir de intereses diferentes.

Finalmente, la presentación de los proyectos de Calcagno y Monasterio ante el Consejo Directivo -el 31 de marzo de 1958- se dio en un marco de gran presión estudiantil. Si bien se trató de dos presentaciones separadas, no eran en realidad proyectos distintos, sino que ponían el énfasis en aspectos complementarios de una misma idea global. Por un lado, era necesario crear una carrera mayor de psicología (con tres especialidades: psicología clínica, laboral y educacional). Por el otro, debían crearse tres carreras menores, que otorgarían el título de auxiliar en cada una de esas especialidades. Calcagno era particularmente optimista respecto de las posibilidades laborales de los futuros graduados: "Por mucho tiempo habrá una gran demanda de psicólogos, y será imposible cubrir todas las plazas ofrecidas en universidades, escuelas, colegios, clínicas, fábricas, talleres, etc." (Facultad de Humanidades y Ciencias de la Educación, 1958, p. 19).

En cuanto a la organización de los estudios, el plan de la carrera de psicología se dividiría en dos ciclos: un primer ciclo de formación básica (compuesto por quince materias agrupadas en tres años) y un ciclo de especialización (con cinco asignaturas teórico-prácticas diferentes para cada especialidad, que podrían cursarse en uno o dos años). El plan del profesorado reemplazaba el ciclo de especialización por una serie de diez materias que completaban la formación científica, pedagógica y filosófica. Tanto la carrera profesional como la docente podrían continuarse a través del doctorado. Para ello era menester cursar cuatro asignaturas complementarias, adscribirse durante un año a un laboratorio o seminario y, finalmente, realizar una tesis. Calcagno, consecuente con su formación, ponía el énfasis en la carrera docente (más larga que la profesional) y en el doctorado (ya que implicaba una especialización en la investigación científica). Las carreras de auxiliares solo se compondrían de seis asignaturas: tres del ciclo básico y tres de la especialidad respectiva.

El informe de Monasterio especificaba el de Calcagno, ahondando en cuestiones teóricas. En su introducción, definía el objeto de la psicología en términos lagachianos, como "el estudio de la conducta y la personalidad". Las diferentes doctrinas psicológicas no representaban otra cosa que "variaciones metodológicas en su acceso al tema exclusivo". Luego de hacer un recorrido por la historia de la psicología en Europa, Estados Unidos y Argentina reivindicaba la competencia exclusiva de la Universidad para la formación de estos nuevos profesionales. Dentro de la Universidad, debía ser la Facultad de Humanidades la encargada de la formación, ya que era la única que podía brindar el abanico "de disciplinas antropológicas, sociales, lingüísticas, pedagógicas y filosóficas" que el acervo del psicólogo requería. Por otra parte, la creación de la carrera le parecía oportuna, ya que se detectaba una alta deserción en las carreras humanísticas más tradicionales respecto de las facultades con carreras técnicas. Así, la carrera de psicología ofrecería una salida profesional atractiva y ventajosa para los estudiantes de ambos sexos, que contribuiría a nivelar el desequilibrio en las inscripciones y proporcionaría a la Facultad "una faceta moderna y dinámica".

En cuanto al campo de actuación del psicólogo, la formación aplicada de Monasterio parecía haber influido en la configuración de un perfil de tipo técnico, donde se destabacan -por lo taxativas-las limitaciones impuestas al psicólogo clínico. Si bien podría ejercer la psicoterapia (definida eufemísticamente como "tratamiento verbal de los conflictos de la personalidad"), solo podría hacerlo bajo la supervisión del médico, por lo que su rol se vería reducido al de un técnico auxiliar, como el del radiólogo o el del bioquímico. No resulta del todo claro si era ese el rol que verdaderamente preveían Monasterio y Calcagno o si se trataba de una formación de compromiso, para evitar la previsible resistencia de la agremiación médica y posibilitar la aprobación del proyecto en el Consejo Superior de la Universidad. En cuanto al psicólogo laboral (especialidad preferida por Monasterio), su campo de acción era 
muy similar al delineado por Watson en Estados Unidos más de cuarenta años antes. Sus funciones tendían a optimizar la relación costo-beneficio de las fábricas y empresas, entre otras cosas, "por la adecuación satisfactoria del hombre a la máquina”. Respecto del psicopedagogo, sus tareas no diferían mucho de las tradicionalmente desempeñadas por los profesores de psicopedagogía y los asistentes educacionales. Se contemplaba además la creación de al menos otras dos especialidades (en psicología jurídica y criminalística, y en psicología social) que jamás se llevaron a la práctica.

Luego de las presentaciones, el debate se centró en dos aspectos: el problema presupuestario que implicaría la apertura de la carrera (cuestión que fue rápidamente resuelta) y el tema del doctorado. Según el proyecto, podría llegarse al doctorado por dos vías distintas: al finalizar la carrera de psicología o al terminar el profesorado. Calcagno había planteado desde un principio que era importante que junto con la creación de la carrera profesional se aprobara el profesorado, porque, de lo contrario, "sería difícil defender en el Consejo Superior un proyecto que parecería puramente técnico" (Facultad de Humanidades y Ciencias de la Educación, 1958, p. 17). Si bien las actas no reflejan el debate, puede leerse entre líneas que en este punto residían las mayores dificultades. Por un lado, era necesario tomar todos los recaudos posibles para que el proyecto, que contaba con un amplio consenso dentro de la Facultad, no naufragara en el Consejo Superior de la Universidad. Pero por el otro, había un velado debate entre Calcagno (acompañado por el establishment de la Facultad) y Monasterio, por el énfasis que se pondría en la investigación y la docencia o en el desempeño profesional.

Finalmente, la creación de la carrera de psicología fue aprobada, pero, pese a las aspiraciones de Monasterio, se privilegió el profesorado, que sería la única vía de acceso al doctorado, ya que "luego de un cambio de ideas, el Consejo Académico resolvió suprimir el doctorado correspondiente a la carrera de psicólogo" (Facultad de Humanidades y Ciencias de la Educación, 1958, p. 25). De este modo, los nuevos psicólogos se verían privados de acceder al máximo título académico (probablemente para evitar la segura oposición de los médicos, a quienes tradicionalmente se les atribuía esa titulación, sin necesidad de estudios adicionales) y solo podrían obtenerlo los profesores, con mayor formación humanística. El tema del doctorado en psicología salía así del área de conflicto profesional con la medicina, ya que pasaba a ser solo una prolongación académica de la carrera docente, que no podía confundirse con el ejercicio de la clínica.

Los diarios locales (El Día y El Argentino) no le dieron gran importancia a la aprobación de este proyecto; sin embargo, La Nación de Buenos Aires le dedicó un par de columnas en las que se reproducía textualmente la presentación de Fernanda Monasterio, y se acotaba que la institución de la carrera de psicología había sido solicitada "por el Centro de Estudiantes de Humanidades y reiterada por estudiantes de distintas carreras, que por nota se dirigieron al decano y al consejo directivo de la Facultad" ("Fue creada en La Plata la Carrera de Psicología", 1958a).

Las semanas siguientes fueron de gran tensión por la presión estudiantil, por lo que el 20 de mayo el Consejo Académico de la Facultad decidió abrir una inscripción provisional que descomprimiera la situación "hasta tanto se apruebe definitivamente la carrera de psicología" (Facultad de Humanidades y Ciencias de la Educación, 1958, p. 84). El día anterior, la Comisión de Enseñanza del Consejo Superior, luego de considerar el proyecto (que incluía el plan de estudios), lo había elevado al Consejo recomendando su aprobación. Finalmente, el 30 de mayo de 1958 el Consejo Superior de la UNLP aprobó la creación de la carrera y la del profesorado y doctorado ${ }^{15}$. Esta vez, pese a que la mayor parte de sus páginas estaba dedicada a los cuartos de final del mundial de Suecia, el principal diario local, El Día, sí reflejaba la novedad:

15 Lamentablemente, no se conservan las actas del Consejo Superior de ese año, por lo que ha sido prácticamente imposible reconstruir con certeza los debates que tuvieron lugar en esa sesión. Los testimonios orales al respecto, dado el tiempo transcurrido, son un tanto confusos y contradictorios, por lo que hemos preferido no basarnos en ellos. No obstante, hay acuerdo en que, paradójicamente, el voto que decidió la votación fue el del consejero Christmann, de la Facultad de Ciencias Médicas, quien pocos meses después sería designado decano de esa casa de estudios. 
Al tratarse sobre la creación de la carrera de psicología, se generalizó en el seno un prolongado debate en el que intervinieron la mayoría de los consejeros, quienes expresaron sus puntos de vista sobre esta carrera. Las opiniones en disidencia se fundamentaron principalmente en el aspecto presupuestario para poner en funcionamiento los mencionados estudios. Tras de escuchar la opinión de los consejeros, la misma fue aprobada. ("Creación de la carrera de psicología”, 1958b)

Es probable que las concesiones realizadas en el proyecto original hayan prevenido una oposición abierta de los representantes de la Facultad de Medicina a partir de argumentos de tipo académico, pero no alcanzaron para impedir los resquemores de todas las facultades por tener que compartir el presupuesto de la Universidad con una "advenediza" (lo cual sería el sino de la carrera de allí en adelante). Restaba ahora organizar las asignaturas del primer año. Con ese fin, se contrató a los profesores Luis María Ravagnan (para dictar Introducción a la Psicología) y Marcelino Tavella (que cumplía funciones técnicas en la Dirección de Psicología Educacional) para el curso de Psicomatemática y Estadística. El elenco se completaba con Juan Cuatrecasas, en Antropología, y Eugenio Pucciarelli y Narciso Pousa en Introducción a la Filosofía ${ }^{16}$. Además, el 17 de junio se contrató a la Dra. Monasterio con un cargo full-time para dictar la asignatura Biología Humana y continuar dirigiendo el Instituto de Psicología. Todo indicaba que Alfredo Calcagno había delegado en ella la función de comandar sus destinos. Dos días después, con gran asistencia de público (en el aula magna de lo que es hoy el Liceo Víctor Mercante), quedaba oficialmente inaugurada con una clase de Biología Humana, previas palabras alusivas de Alfredo Calcagno ${ }^{17}$.

16 Narciso Pousa terminó a cargo de las clases para la carrera de psicología. Al igual que Pucciarelli, tenía una amplia formación en filosofía contemporánea, comentando autores como Bergson y Nietzsche o Heidegger y Jean Wahl (cuyos cursos de metafísica había seguido en París durante 1952 y 1953).

17 Tres meses después, Calcagno sería nombrado Embajador Extraordinario y Ministro Plenipotenciario ante la UNESCO, con sede en París, de donde solo regresaría poco antes de su muer-

\section{Comentarios finales}

Cuando el Consejo Superior de la UNLP aprobó la creación de la carrera, el decano que había impulsado el proyecto inicial, Bernardo Canal Feijóo, ya había dejado su puesto en manos del decano electo según los nuevos estatutos. A su vez, Alfredo Calcagno se aprestaba a partir a París, donde se desempeñaría como embajador argentino ante la UNESCO, hasta su muerte, en 1962. Pero antes de marcharse, según vimos, ya había ungido a Fernanda Monasterio como su sucesora. En efecto, ella había sido contratada de tiempo completo para dictar la asignatura Biología Humana y para seguir dirigiendo el Instituto. Además, en 1959, al crearse el Departamento de Psicología, ella fue designada como su responsable. Todo indicaba que la carrera de La Plata, a diferencia de lo que sucedía en el resto del país, iba a orientarse hacia una psicología científica, de corte experimental, heredera de la tradición inaugurada por Mercante y desarrollada por Calcagno. Sin embargo, en la Facultad de Humanidades, al igual que en el país en su conjunto, el clima de ideas parecía ir más bien en otra dirección. Tan es así que, en 1964, Monasterio se vería obligada a renunciar a la jefatura del Departamento, siendo reemplazada por Luis María Ravagnan, quien representaba una tradición filosófica y humanística mucho más afín al recorrido de Canal Feijóo.

En realidad, todo hace pensar que el proyecto de Calcagno y Monasterio de recrear en La Plata una tradición científica (sino experimental, al menos de orientación objetiva), en muy pocos años, terminó naufragando. Más allá de las intenciones y las características personales de la médica española (que hizo todo lo que estuvo a su alcance para llevar ese programa a la práctica), se encontró con condiciones adversas en distintos planos. En primer lugar, había limitaciones de tipo material, lo cual se hizo evidente ya en 1960, cuando trató de revitalizar el Instituto de Investigación con los res-

te. Por lo tanto, desde la dirección del Instituto de Psicología, Monasterio quedó en los hechos al frente de la carrera (aunque Calcagno, desde Francia, seguiría siendo su figura de consulta y referencia). 
tos de los laboratorios experimentales creados por Calcagno y Jakob a principios de siglo, que habían sido desmantelados durante el peronismo. Con ese fin, debió encargar en Europa aparatos tales como un taquistoscopio electrónico, un reactómetro, un cronoscopio y un aparato de Giese-Lahy para medir la atención concentrada (Monasterio, 1961, p. 8). Aunque los aparatos llegaron ese mismo año, la contratación de un profesor para Psicología Experimental resultó menos sencilla. El profesor elegido en 1959 había tenido que renunciar, a causa del descontento de los estudiantes, que habían boicoteado su curso (N. Delucca, comunicación personal, noviembre 10,1998). Ante este obstáculo, Alfredo Calcagno, que aún estaba en París, logró convencer a su amigo Emilio Mira y López para suplir en parte esa carencia. De modo que Mira viajó a La Plata para dar un curso sobre "psicología de la expresión motriz" y una serie de conferencias sobre "las direcciones de la psicología actual" y "la psicotécnica en orientación profesional" (Monasterio, 1961, pp. 8-9).

Es decir, que a las limitaciones materiales debía agregarse la escasez de recursos humanos formados, que a su vez se sumaba a la dificultad de la joven profesora para conciliar su propio proyecto con las expectativas de los estudiantes. Por ejemplo, en 1961, convocó a Adolfo Lértora, un psiquiatra comunista (de la línea más dura del partido) que trabajaba en el Hospital Psiquiátrico de Melchor Romero, para ocupar la cátedra de Psicopatología General. Aparentemente, Monasterio había tratado de ubicar en esa materia clave de la rama clínica a alguien cercano a su propia concepción naturalista del psiquismo. Sin embargo, había escogido a un psiquiatra reflexólogo que no solo había impugnado ácidamente a José Bleger (el principal referente de los alumnos de Buenos Aires y Rosario, así como el creador de una nueva psicología de filiación psicoanalítica con vocación social), sino que también había definido la psicología como "la teoría y la práctica de la ingeniería del alma juvenil progresista", por oposición a la "recíproca masturbación mental psicoanalítica" (Lértora, 1959, p. 334). No hace falta decir que ese tipo de afirmaciones no concitaron la adhesión de los estudiantes, que también boicotearon el curso de ese psiquiatra hasta obligarlo a renunciar $(\mathrm{N}$. Delucca, comunicación personal, noviembre 10, 1998).

Sin embargo, las elecciones de Monasterio no siempre estuvieron orientadas en ese sentido. En 1960, por ejemplo, para la cátedra de Neurobiología, había llamado a Edgardo Rolla (1910-2001), un neurocirujano formado con Ramón Carrillo. A principios de los años cincuenta, durante su especialización en los Estados Unidos, Rolla también se había encontrado con el psicoanálisis, convirtiéndose luego en discípulo de Pichon-Rivière y en miembro adherente de la APA. En 1961, se iba a hacer cargo además de la materia Psicología Profunda, mientras escribía su libro Psicoterapia individual y grupal, que iba a ser publicado por Paidós el año siguiente. Asimismo, para ser reemplazada en Psicología de la Infancia y de la Adolescencia, Monasterio había convocado a Maurico Knobel (1922-2008), un joven médico especializado en psiquiatría infantil en Missouri, en la Greater Kansas City Mental Foundation, entre 1956 y 1959. Ya en 1955, Knobel había sido nombrado vicepresidente para América del Sur de la Sociedad Interamericana de Psicología (SIP). Lo que Monasterio no sabía era que, mientras enseñaba su materia, Knobel también iba a iniciar su formación psicoanalítica en la APA, en 1961, convirtiéndose en miembro adherente ese mismo año. Según el testimonio de la fundadora:

La gente de La Plata no era psicoanalítica, ni sabía nada del tema hasta que llegaron algunos profesores como Knobel y Rolla -que eran psicoanalistas, pero no fueron a enseñar psicoanálisis, porque Rolla fue a explicar neurobiología-. Yo le dije - "aquí no metas psicoanálisis, sino el cerebelo y el bulbo raquídeo". "Allí tienes los preparados de la época de Christofredo Jakob". (Monasterio, comunicación personal, septiembre 5, 1997)

Más allá del voluntarismo de esas expresiones, dentro de la propia Facultad de Humanidades, es claro que, ya desde los años veinte, el clima de ideas no era afín a ese tipo perspectivas. Y lo sería aún menos a principios de los años sesenta. Por otra parte, los alumnos de la carrera, que en un principio 
tomaron a Monasterio como su adalid (en razón de su juventud, por la firmeza con la que defendía tanto sus ideas como el espacio institucional a su cargo y hasta su propia orientación sexual), rápidamente se fueron apartando de sus formas de concebir la psicología. Aunque en un principio la consideraran como a una militante de izquierda exiliada del franquismo (cosa que la propia Monasterio siempre desmintió), esa supuesta militancia no alcanzaba para que sus propios intereses intelectuales (que iban desde el existencialismo hasta el psicoanálisis) no entraran en conflicto con el naturalismo a ultranza de la joven profesora. Ella, a su vez, no llegaba a comprender hasta qué punto las nuevas ideas que se implantaban por la vía cultural (y no solo por la académica) modelaban las aspiraciones de los estudiantes y también las de muchos profesores.

A lo largo de esos años, se había dado entre los alumnos de las primeras promociones y sus profesores una relación muy particular. Los "combates" por la carrera (tanto dentro de la Facultad como con el campo médico por la defensa de las incumbencias en el campo de la clínica) habían contribuido a forjar una identidad que los aunaba como "compañeros de lucha". Se generó un trato casi familiar, al punto de que periódicamente se organizaban reuniones en Buenos Aires, en las casas de algunos profesores, en las que se cenaba y se charlaba de temas culturales o políticos (O. Ferreiroa, comunicación personal, julio 15, 1997). La capital parecía estar cada vez más cerca, y también por esa vía, contra todas las previsiones, el psicoanálisis había entrado finalmente en la carrera. Se hacían frecuentes los viajes nocturnos para hacer cursos con Pichón-Rivière, Edgardo Rolla o el mismo Ángel Garma, que complementaban la formación obtenida en las materias del plan de estudios. La Psicología de la conducta, de Bleger, se había transformado en libro de referencia obligada (Bleger, 1963). Muchos de los alumnos habían comenzado su análisis personal, generalmente con Emilio Dupetit (adjunto de Psicología Profunda), con su esposa, Angélica de Dupetit, Edgardo Rolla o Marie Langer. Incluso algunos, aquejados por los altos honorarios del tratamiento individual, accedieron a la terapia de grupo y al psicodrama con el grupo de Eduardo Pavlovsky (quien ya había dado una serie de conferencias en La Plata). Hacia el final de la carrera, la mayoría de las materias aplicadas del área clínica remitían, de un modo u otro, a las categorías del psicoanálisis. Según otra alumna de la primera promoción,

No me caben dudas de que ya, por entonces, la propuesta del psicoanálisis era la más rica, sistematizada y coherente. La que nos aportó un conocimiento o una interrogación nueva sobre el ser humano. Pero, a diferencia de lo que puede pasar hoy, era una propuesta no alienada, que incluía otros atravesamientos. Teoría de los grupos, teoría de la comunicación, aportes de la filosofía existencial, y hasta el estudio del funcionamiento neuroendocrino y los efectos de los psicofármacos. (Delucca, 1994)

En el heterogéneo mosaico de saberes que habían intervenido en la formación de los estudiantes, el paradigma experimental que regía las asignaturas del ciclo básico había dado paso rápidamente a una síntesis ecléctica -de la que el psicoanálisis parecía ser la matriz-donde intervenían la fenomenología (sobre todo Merleau-Ponty), el existencialismo (Sartre y Binswanger) y la reflexología, pero también la psicología en lengua francesa (Piéron, Wallon, Lagache y, por supuesto, Piaget).

Cada vez más, los estudiantes reclamaban que el psicoanálisis, tan prestigiado en la cultura, tuviera un lugar acorde en la carrera. Los nuevos alumnos que iban ingresando, menos comprometidos que los primeros con las "batallas fundacionales", no tenían por Monasterio -ni por su línea teórica- la admiración y el respeto que en cierta medida había inspirado en aquellos. Luego del fallecimiento de Calcagno, y electo un nuevo decano en reemplazo de Rossenvaser, la doctora española había perdido a sus mayores aliados, y no tenía muchos recursos para seguir sosteniendo una tradición experimental cuya hegemonía parecía cada vez más inviable. Extrañamente, a principios de 1964, llegó al Consejo Académico de la Facultad la propuesta de que Monasterio fuera elevada a la categoría de "Profesora Extraordinaria" (distinción que muy pocos docentes de la casa habían obtenido hasta entonces). Como era de esperar, la moción fue rechazada por una 
abrumadora mayoría de ocho votos en contra, dos abstenciones y solo dos votos a favor ${ }^{18}$. Casi inmediatamente, Monasterio renunció a su cargo como jefe del Departamento y directora del Instituto, y se mudó a Buenos Aires. Seguiría dictando su cátedra hasta 1966 y luego regresaría a España. En su lugar, en marzo de 1964 se designó a Luis María Ravagnan, un filósofo amante de Merleau-Ponty y versado en una psicología más cercana de la filosofía que de la ciencia.

Ravagnan estuvo al frente del Departamento por poco más de un año, período durante el cual su logro más importante fue la creación de la Revista de Psicología, que tendría cierta periodicidad hasta 1967, con la edición de cinco números ${ }^{19}$. Ravagnan, que naturalmente fue su director, en su presentación hablaba de una apertura "a todas las trayectorias en las que esté comprometido el hombre". En agosto de 1965, luego de su jubilación, fue sucedido al frente del Departamento y la revista por el médico Juan Carlos Pizarro. En los años cuarenta, Pizarro había iniciado su análisis didáctico en la APA con Arnaldo Rascovsky, y supervisaba con Ángel Garma. Luego de suspender su tratamiento con Rascovsky, se vio obligado a abandonar el Instituto. Sin embargo, continuó analizándose con Marie Langer, quien lo aceptó como paciente. Luego de abandonar también ese tratamiento, inició un último análisis con Emilio Rodrigué. Prosiguió su carrera como psicoterapeuta fuera de la APA. Llegó a ser jefe de psiquiatría en el Hospital de Niños de Buenos Aires y participó en la redacción de la Ley Penitenciaria Nacional, gracias a un cargo jerárquico que obtuvo por concurso en la Dirección de Institutos Penales (Balán, 1991). Luego se especializó en Rorschach y asíllegó a ser titular de Psicodiagnóstico en la carrera de psicología, donde se ganó el respeto de la mayoría de los alumnos, que lo consideraban un excelente profesor. Con Mauricio Knobel al frente del Instituto, Pizarro seguiría como jefe de Departamento

18 De esos dos votos, uno era el de José María Lunazzi, discípulo dilecto y heredero de Calcagno.

19 En esta primera época, seguiría apareciendo de manera muy discontinua, con un número en 1973, otro en 1979, uno más en 1981 y una última entrega en 1983. hasta la intervención de 1973, incluso bastante más allá del golpe de estado de 1966, que en otras carreras del país supuso renuncias masivas, cuando no la expulsión de numerosos profesores (como José Bleger o Jaime Bernstein). En todo caso, ese quiebre institucional marcó el fin de un período de franca expansión de la psicología, que en adelante (y sobre todo después del golpe de 1976) comenzaría a ser cada vez más renuente a apropiarse de una escena pública que se hacía cada vez más peligrosa. De este modo, la actividad clínica privada, desarrollada en consultorio, tendió a expandirse cada vez más, contra las expectativas de los propios fundadores e incluso al margen de las regulaciones legales (que reservaban a los médicos cualquier tipo de actividad psicoterapéutica). Que en 1966, en la carrera de psicología de la UNLP, el director del Instituto y el jefe del Departamento fueran un psicoanalista y un psicoterapeuta, respectivamente, estaba perfectamente en consonancia con los nuevos tiempos.

No obstante, esta "privatización" del ejercicio profesional del psicólogo (que cada vez más se acercaba a la figura del psicoanalista) no solo implicó el fracaso del proyecto de Calcagno y Monasterio, sino también el de propuestas como las de Bleger, Ulloa, Rolla y otros discípulos de Enrique Pichon-Rivière, quienes creían en un psicoanálisis operativo, que sirviera como herramienta para el cambio social. En última instancia, lo sucedido en La Plata en los años sesenta, no podría entenderse cabalmente sin inscribirlo en un contexto más amplio, de alcance nacional, en el que se produjo un proceso de clinicización de la disciplina y de asimilación del rol del psicólogo al del psicoanalista.

\section{Referencias}

Balán, J. (1991). Cuéntame tu vida: una biografía colectiva del psicoanálisis argentino. Buenos Aires: Planeta.

Biagini, H. (1995). La huelga grande de La Plata. Todo es historia, 330, 17-35.

Bleger, J. (1963). Psicología de la conducta. Buenos Aires: Eudeba.

Calcagno, A. (1921). Laboratorios de la Facultad de Ciencias de la Educación. Humanidades, 1, 580 596. 
Canal Feijóo, B. (1934). Nivel de historia y otras proposiciones. Santiago del Estero: Nan.

Canal Feijóo, B. (1937). Ensayo sobre la expresión popular artística en Santiago del Estero. Buenos Aires: Compañía Impresora Argentina.

Canal Feijóo, B. (1938). Mitos perdidos. Buenos Aires: Compañía Impresora Argentina.

Canal Feijóo, B. (1943). La expresión popular dramática. San Miguel: Facultad de Filosofía y Letras de la UNT.

Canal Feijóo, B. (1951). Teoría de la ciudad argentina. Idealismo y realismo en el proceso constitucional. Buenos Aires: Sudamericana.

Canal Feijóo, B. (1955). Juan Bautista Alberdi: constitución y revolución. Buenos Aires: Fondo de Cultura Económica.

Carpintero, H. (2012). Fernanda Monasterio, una voz española en la Sociedad Interamericana de Psicología (SIP). Revista Interamericana de Psicología/ Interamerican Journal of Psychology, 46(1), 43-50.

Cuerpo docente: curso de 1945. (1945). Humanidades, $32,236-237$.

Creación de la carrera de psicología. (1958b, 5 de junio). El Día.

Dagfal, A. (1996). Alfredo Calcagno: pedagogía científica y psicología experimental. Cuadernos Argentinos de Historia de la Psicología, 2(1-2), 109-123.

Dagfal, A. (2009). Entre París y Buenos Aires. La invención del psicólogo (1942-1966). Buenos Aires: Paidós.

Dagfal, A. (2011). Entrevista a la Dra. Fernanda Monasterio Cobelo (1920-2006). Revista de Historia de la Psicología, 32(4), 37-64.

Dagfal, A. (2012). Psychoanalysis in Argentina under peronism and anti-peronism (1943-1963). En J. Damousi \& M. Plotkin (Comps.), Psychoanalysis and politics: Histories of psychoanalysis under conditions of restricted political freedom (pp. 135-163). Nueva York: Oxford University Press.

Delucca, N. (1994). Palabras de una veterana de la primera promoción de psicólogos a los estudiantes y futuros colegas. Buenos Aires: Universidad Nacional de La Plata.

Facultad de Humanidades y Ciencias de la Educación. (1954). Actas del Consejo Directivo. Buenos Aires: Universidad Nacional de La Plata.
Facultad de Humanidades y Ciencias de la Educación (1958). Actas del Consejo Directivo. Buenos Aires: Universidad Nacional de La Plata.

Fue creada en La Plata la Carrera de Psicología. (1958a, abril 2). La Nación.

García Sebastiani, M. (2005). Los antiperonistas en la Argentina peronista. Radicales y socialistas en la política Argentina entre 1943 y 1955. Buenos Aires: Prometeo.

Gentile, A. (2003). Ensayos históricos sobre psicoanálisis y psicología. Rosario: Fundación Ross.

Klappenbach, H. (2001). La Psicología en Argentina: 1940-1958. Tensiones entre una psicología de corte filosófico y una psicología aplicada. Tesis doctoral, Facultad de Filosofía y Letras, Universidad de Buenos Aires.

Kurowsky, M. (2001). La obra psicológica de Juan Cuatrecasas Arumí (1899-1990). (Tesis doctoral). Universidad Complutense de Madrid, Madrid, España.

Lértora, A. (1959). Psicoanálisis y dialéctica materialista. Acta Neuropsiquiátrica Argentina, 5, 334-339.

Lunazzi, J. M. (1965). Alfredo D. Calcagno, pasión educadora, valor cívico. La Plata: Universidad Nacional de La Plata.

Mercante, V. (1927). La paidología. Estudio del alumno. Buenos Aires: M. Glazer.

Monasterio, F. (1961). Memorias del Departamento de Psicología. La Plata: Universidad Nacional de La Plata.

Munín, H. (1989). La Dirección de Psicología de la Provincia de Buenos Aires: cuarenta años a la búsqueda de su lugar (Informe de investigación). Buenos Aires: CONICET - Ciencias de la Educación.

Piñeda, A. (2006). La psicología neoescolástica experimental en Argentina y España: 1920-1960. San Luis, Ar: Universidad Nacional de San Luis, Facultad de Ciencias Humanas.

Plotkin, M. (2003). Freud en las pampas. Buenos Aires: Sudamericana.

Pronko, M. (1995, septiembre). Peronismo y universidad: una mirada histórica. Reflexiones en torno de la Ley 13031 de 1947. Trabajo presentado en el Primer Encuentro Nacional, La universidad como objeto de investigación, UBA, Argentina.

Quintana, J., \& Feldman, R. (2007). Apunte biográfico sobre F. Monasterio. Boletín informativo SEHP, $39,5-15$. 
Talak, A. M. (2007). La invención de una ciencia primera. Los primeros desarrollos de la psicología en la Argentina (1896-1919). (Tesis doctoral). Facultad de Filosofía y Letras de la Universidad de Buenos Aires, Argentina.

Tasso, A. (1997). Biografía y clima de época en el pensamiento de un creador. ¿Quién fue Bernardo Canal Feijóo? Santiago del Estero: Barco.

Terán, O. (1991). Nuestros años sesentas. Buenos Aires: Puntosur.

Vallejo, P. (2008). El psicoanálisis de Bernardo Canal Feijóo. Historia y vocación. (Tesis doctoral, publicada en 2010 con el título Bernardo Canal Feijóo en la historia del psicoanálisis en la Argentina). Uni- versidad Nacional de Tucumán, San Miguel de Tucumán, Argentina.

Universidad Nacional de La Plata (1954). Dictamen de las Comisiones de Interpretación y Reglamento y Enseñanza del Consejo Superior de la Universidad Nacional de La Plata (7/6/54, exp. He. 3869/54). Buenos Aires: Autor.

Vezzetti, H. (1996). Aventuras de Freud en el país de los argentinos. Buenos Aires: Paidós.

Wagner, E., \& Wagner D. (1934). La civilización chacosantiagueña y sus correlaciones con las del Viejo y Nuevo Mundo (B. Canal Feijóo, Trad.). Buenos Aires: Compañía Impresora Argentina. 
